\title{
Development of a NIR-VIS-UV lidar echo emulator
}

Monica Rodríguez Cortina, Pawel Adamiec, Juan Barbero, Xabier Quintana, Morten Andreas Geday

Monica Rodríguez Cortina, Pawel Adamiec, Juan Barbero, Xabier Quintana, Morten Andreas Geday, "Development of a NIR-VIS-UV lidar echo emulator," Proc. SPIE 11852, International Conference on Space Optics - ICSO 2020, 118526F (11 June 2021); doi: 10.1117/12.2601249

SPIE Event: International Conference on Space Optics - ICSO 2021, 2021, Online Only 


\section{International Conference on Space Optics-ICSO 2020}

Virtual Conference

30 March-2 April 2021

Edited by Bruno Cugny, Zoran Sodnik, and Nikos Karafolas
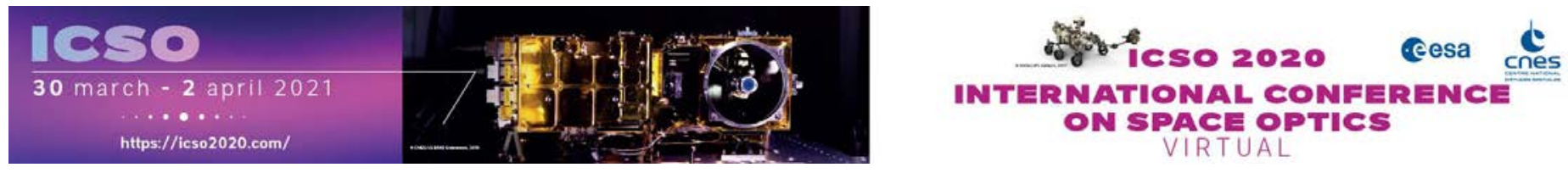

Development of a NIR-VIS-UV lidar echo emulator

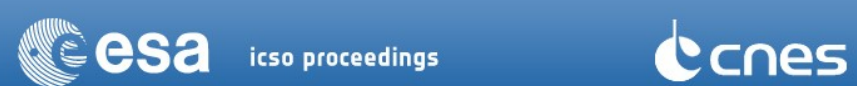

International Conference on Space Optics - ICSO 2020, edited by Bruno Cugny, Zoran Sodnik, Nikos Karafolas, Proc. of SPIE Vol. 11852, 118526F - (c) 2021 ESA and CNES

CCC code: $0277-786 \mathrm{X} / 21 / \$ 21 \cdot$ doi: $10.1117 / 12.2601249$ 


\title{
Development of NIR-VIS-UV LIDAR Echo Emulator
}

\author{
Mónica Rodríguez Cortina*a,b, Pawel Adamiec ${ }^{\mathrm{a}}$, Juan Barbero a , Xabier Quintana ${ }^{\mathrm{b}}$, Morten Andreas \\ Geday $^{\mathrm{b}}$
}

\author{
aALTER TECHNOLOGY TÜV NORD S.A.U., C/ la Majada 3, 28760 Tres Cantos, Spain; \\ ${ }^{\mathrm{b}}$ CEMDATIC, ETSI Telecomunicación, Universidad Politécnica de Madrid, Av. Complutense 30, \\ 28040 Madrid, Spain;
}

\begin{abstract}
Near-infrared, visible and ultraviolet (NIR-VIS-UV) lidar echo emulator (LEE) was developed to emulate the returns of a spatial lidar at three wavelengths $(355 \mathrm{~nm}, 532 \mathrm{~nm}$ and $1064 \mathrm{~nm})$ within the framework of the HOLDON project. It is used to characterize the detection chain formed by a $\mathrm{HgCdTe}$ avalanche photodiode (MCT APD), read-out integrated circuit (ROIC), and surrounding electronics, which will be used in a lidar remote sensing of earth's atmosphere. It emulates two echoes in the three wavelengths. A short echo (pulse width $<10 \mathrm{~ns}$ ) with optical power dynamic range between $0.2 \mathrm{nW}$ and $200 \mathrm{nW}$ and a long echo (pulse width of about $270 \mu \mathrm{s}$ ) in range from $0.1 \mathrm{pW}$ to $25 \mathrm{pW}$. The long echo has two adjustable parameters which allow changing the shape of the pulse (from a ramp to a rectangular pulse). The combination of both echoes allows characterizing the detector over the full $60 \mathrm{~dB}$ dynamic range, the target for the detection chain. The system consists of only one laser diode at $1064 \mathrm{~nm}$, a driver, and an arbitrary wave generator (AWG) as seed to create the three waveleghts. The second harmonic generation (SHG) technique was used to generate the beam at $532 \mathrm{~nm}$ from the $1064 \mathrm{~nm}$ laser diode. In this case, a non-linear KTP crystal was used. The sum frequency generation (SFG) technique was used to generate the beam at $355 \mathrm{~nm}$ from both previous beams. For the SFG, two Periodic Poled Lithium Niobate (PPLN) crystals were used. To optimize the output at $355 \mathrm{~nm}$ the temperature of the PPLN crystals was controlled by means of Peltier elements. A LabView program was designed to control all the configuration parameters of both echoes (long and short) and the laser. The software controls also the optimal temperatures where the highest power is reached at $355 \mathrm{~nm}$. The overall system was assembled as an elegant breadboard for easy transportation. The NIR-VIS-UV LEE offers all the necessary requirements for exhaustive characterization of the lidar detection chains at three wavelengths and high dynamic range.
\end{abstract}

Keywords: emulator, lidar, HgCdTe APD, lidar return, pulse shaping, dynamic range, SFG, SHG.

\section{INTRODUCTION}

The advances in MCT APD detectors at the beginning of this century have paved the way to new applications in which detector sensitivity plays an important role. This is the case of lidar systems developed for space where the sensitivity of the detector determines the power of the emitting laser and the diameter of the receiving telescope. Therefore, reducing these two factors would reduce the size and the cost, important factors considered in the development of space lidars [1]

The main features of MCT APD are high quantum efficiency, large electrical bandwidth, and its capability to extend these performances to visible and even UV wavelengths [2]. This technology is currently the best option to build a high performance and versatile lidar detection chain for space. Hence, MCT APD detectors for atmospheric lidar are being currently developed within the framework of Research and Technology National Centre for Space Studies (R\&T CNES) projects and a H2020 project called HOLDON. The objective of these projects is to optimize characteristics of the MCT APD detector modules to reach the specific challenges imposed by atmospheric lidar measurements in terms of dynamic range, response time, temporal lag (remanence) and background noise [3].

The exhaustive characterization of the lidar detection module is an important step to carry out such optimization. For this purpose, a device capable of emulating the return signals of a space lidar in the near infrared was developed by ALTER TECHNOLOGY and was called a near-infrared lidar echo emulator (NIR-LEE) [4].

*monica.rodriguez@altertechnology.com 
It was later delivered to AIRBUS to characterize two detector modules developed by CEA/Leti. The results obtained from the first characterization (using the NIR-LEE) in AIRBUS showed that all the characterized parameters met the specifications, except the quantum efficiency to excess noise factor ratio (QEFR) and the remanence (time delay induced by a strong signal) [3]. These and other critical optical parameters will be explored in the frame of the H2020 HOLDON project [5].

In-depth characterization of the lidar detection module is a key task to evaluate compliance with the requirements for which it was designed and to feed future designs with real figures from the lidar detection chain. For this purpose, a novel LEE was designed and built, allowing emulation of a UV to NIR lidar echoes as seen from space, in terms of optical signal dynamics and temporal features (e.g. duration, transitions, and repeat cycle). In this paper we present the NIR-VIS-UV LEE which will be used in laboratory environment in order to characterize the most critical parameters of the lidar detection module (a MCT APD directly hybridized with a custom designed CMOS ROIC). In the next sections, the design, development, and characteristics of the echoes will be described in detail.

\section{REQUIREMENTS OF LIDAR ECHOES}

The features of the lidar echoes that the system must emulate to characterize the MCT APD are shown in the Figure 1. The echoes have following characteristics:

- $\quad$ short echo with pulse width $<10 \mathrm{~ns}$ and adjustable peak power between 0.2 to $200 \mathrm{nW}$.

- long echo with pulse width $=270 \mu$ s (corresponds to the time necessary to travel through the atmosphere) and adjustable peak power between 0.1 to $25 \mathrm{pW}$.

The shape of the long echo can be modified by means of two parameters: $\alpha$ and $\beta . \alpha$ is a percentage related to the initial current that flows through the diode laser at time $t=0 \mathrm{~s}$ and $\beta$ is a percentage related to the time that long echo has the maximum peak power. For example, in the Figure 1 the values $\alpha$ and $\beta$ are set to $30 \%$ and $50 \%$, respectively. As it can be seen, the short echo is much more powerful than the long echo and one of the challenges is to generate a power drop between them that can reach $63 \mathrm{~dB}$, which is the difference between the maximum power of the short echo $(200 \mathrm{nW})$ and minimum power of the long echo $(0.1 \mathrm{pW})$.
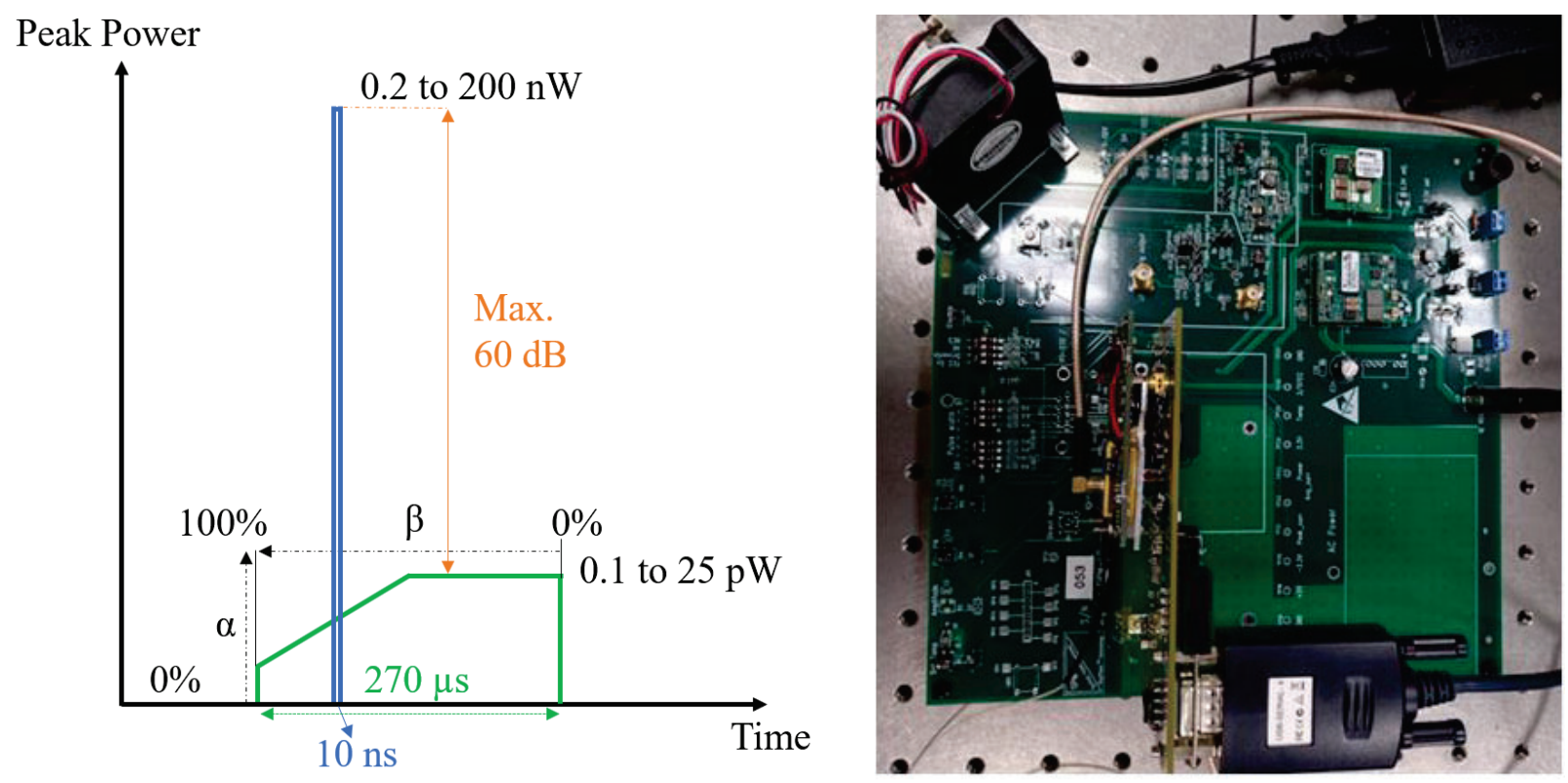

Figure 1. Requirements (left) and OPGS (right). 


\section{SYSTEM DESCRIPTION}

The block diagram of the complete system can be seen in the Figure 2. It consists of 4 different parts: optical pulse generator system (OPGS), NIR stage, VIS stage and UV stage. Each part that makes up the system will be described below.

\subsection{Optical pulse generator system}

The optical pulse generation system is formed by arbitrary waveform generator (AWG), high bandwidth linear current driver, efficient TEC controller and an integrated laser diode (LD). Due to the required pulse width of the short pulse, a wave generator with a minimum clock of $0.1 \mathrm{GHz}$ is necessary. For this purpose, the OPM-LD-ps module from the manufacturer Optical pulse machines (OPM) was acquired to generate both the short and long pulse. This module is a "sandwich" of two boards: the arbitrary wave generator and a high current (HC) electrical-to-optical (EO) converter board (model OPM-LD-Lin-HC). The AWG's clock rate is $1.8 \mathrm{GHz}$ which enables generation of waveforms in subnanosecond resolution. The HC-EO converter board allows a laser peak current up to $2 \mathrm{~A}$. This emulator works with a laser diode (LD-1064-PM-p1200, Innolume). The mean wavelength is $1064.372 \mathrm{~nm}$ and FWHM is $3.264 \mathrm{~nm}$ (conditions: 1\% duty cycle, 500 ns pulse width, pulse operation). The peak power is $1.2 \mathrm{~W} @ 2 \mathrm{~A}$ and threshold current is $61 \mathrm{~mA}$. The type of optical fiber is PANDA PM980 (the output light is polarized along the slow axis of PM fiber), the core diameter is $5.5 \mu \mathrm{m}$ and the mode-field diameter (MFD@1064 nm) is $7.2 \mu \mathrm{m}$.

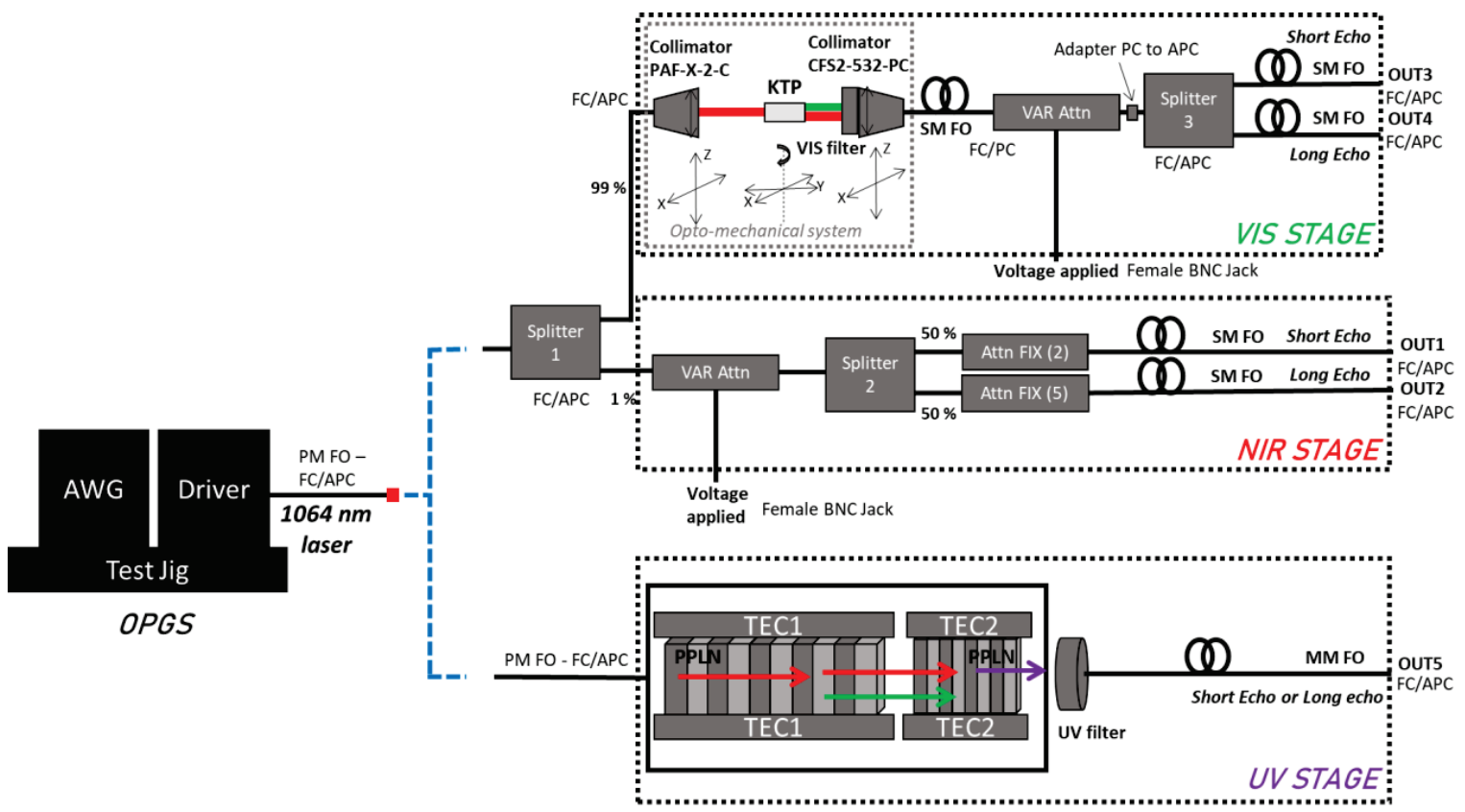

Figure 2. Block diagram of the complete system.

The maximum number of samples to build the waveform is 55,200. Since the AWG is clocked at $1.8 \mathrm{GHz}$, each sample corresponds to $0.5556 \mathrm{~ns}$. Therefore, a short pulse of $10 \mathrm{~ns}$ consists of 18 samples. The long echo with the pulse width of $270 \mu$ s cannot be generated directly from one set of samples due to its maximum length of $30.669 \mu$ s. Fortunately, the OPM-LD-ps provide six multipliers (x1, x2, x4, x8, x16 and x32) which can be used to extend the pulse duration. For $270 \mu \mathrm{s}$ pulse width a multiplier of 16 was used. The long pulse was generated with repetition rate in the $100-500 \mathrm{~Hz}$ range. This means that the duty cycle must be adjustable between $2.7 \%$ to $13.5 \%$.

A program was created using the LabVIEW environment (see Figure 3) that allowed to configure the shape of the pulses, pulse width, period, duty cycle, bias level, trigger source, laser temperature, control low-pass filter configuration, and also enabled to display the important parameters of the laser diode in a LCD screen. 
The generated optical power feed either the UV generation system or the VIS - NIR stages. The optical power from the laser at $1064 \mathrm{~nm}$ is distributed in two channels by means of an optical coupler (TN1064R1A1B, Thorlabs). In the first channel, $99 \%$ of the optical power is used to generate the SHG in the VIS stage and both echoes. The rest of the optical power $(1 \%)$ from the second channel is used to generate the short or long echo in the NIR stage. The details will be described in the following sections.

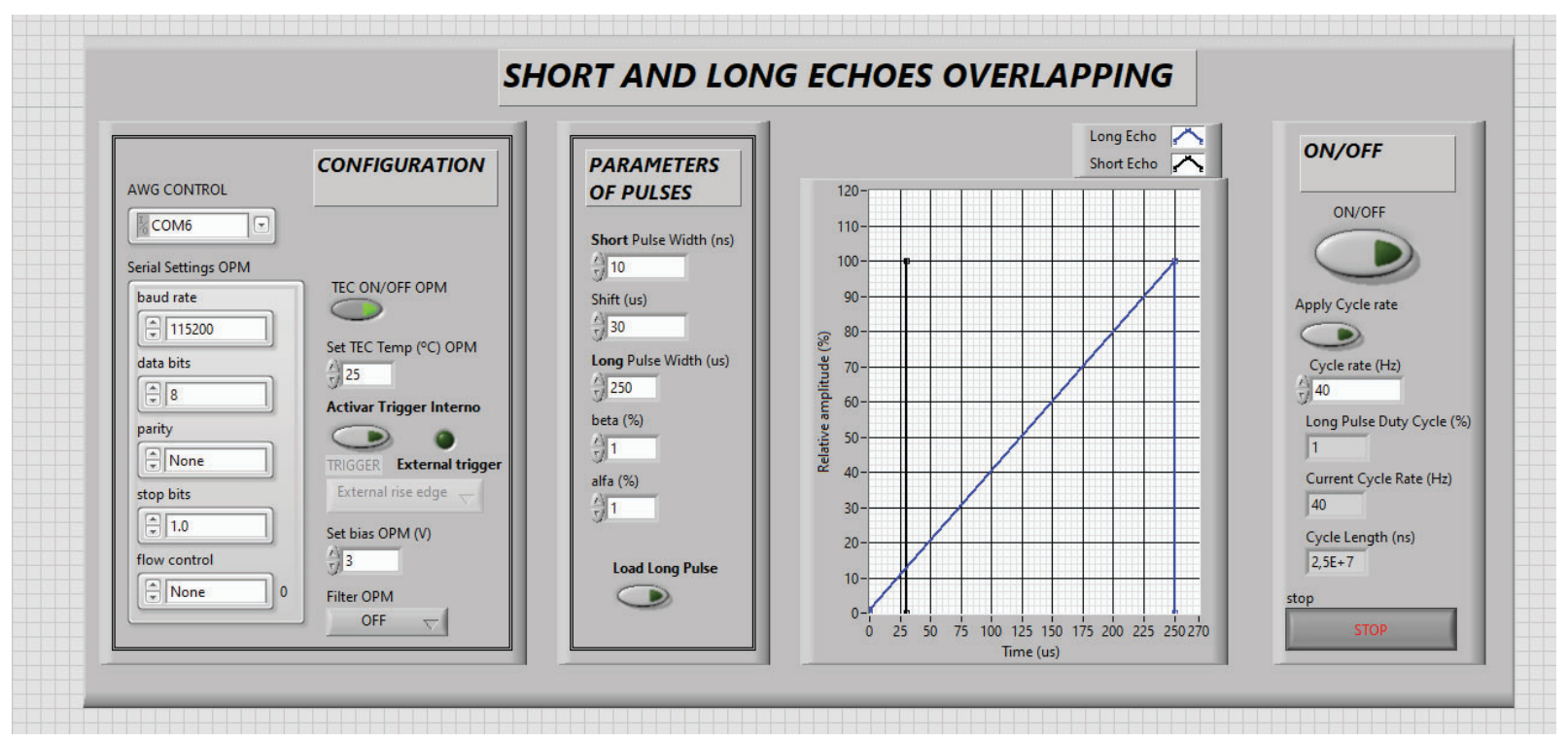

Figure 3. Frontend of the developed software to control the LEE.

\subsection{Near-infrared stage}

In the second channel (after the coulpler along the $1 \%$ path), a voltage-controlled optical attenuator (V1000A, Thorlabs) was placed. Its working principle is as simple as applying a voltage within the range from 0 to $5 \mathrm{~V}$ which is proportional to the attenuation from 0 to $45 \mathrm{~dB}$. Variable attenuator allowed easily adjusting the power levels for both echoes between their maximum and minimum values.

To obtain the echoes, a 50:50 optical coupler (PMC-001-T-10-50-FC/APC, General Photonics) was used to divide power into two equal parts. One branch was used to generate the short echo and the other branch was used to generate the long echo. Then, a set of fixed attenuators were used to reach the peak power levels required by the project. A maximum level of $200 \mathrm{nW}(-37 \mathrm{dBm})$ and a minimum level of $0.2 \mathrm{nW}(-67 \mathrm{dBm})$ were required for the short echo, and for the long echo a maximum level of $25 \mathrm{pW}(-76 \mathrm{dBm})$ and a minimum level $0.1 \mathrm{pW}(-100 \mathrm{dBm})$. The peak power after 50:50 coupler output was meaused and depend on the obtained values, fixed attenuators were placed until the maximum level was reached. Once the maximum value was obtained, the variable attenuator was used to get any power between the maximum and the minimum level. In case of short echo branch, three fixed attenuators (two FA15T and one FA20T) were used and five (FA20T) in the long echo one. At the echo both signals can be merged by means of an additional 50:50 coupler.

\subsection{Visible stage - Second Harmonic Generation (SHG)}

As aforementioned, the $99 \%$ of the optical power was used to generate the SHG, which was achieved through a nonlinear crystal. The chosen crystal was a potassium titanyl phosphate (KTP). This nonlinear crystal is characterized by high nonlinear conversion coefficient, wide acceptance angle, high-allowed temperature, small walk-off angle, and relatively high damage threshold [6]. Also, it has been widely applied to generate the SHG green light, mainly, in solid state lasers (for example, neodymium-YAG laser). The dimensions of the chosen KTP are $3 \times 3 \times 10 \mathrm{~mm}$ (KTP402, Eksma Optics). 

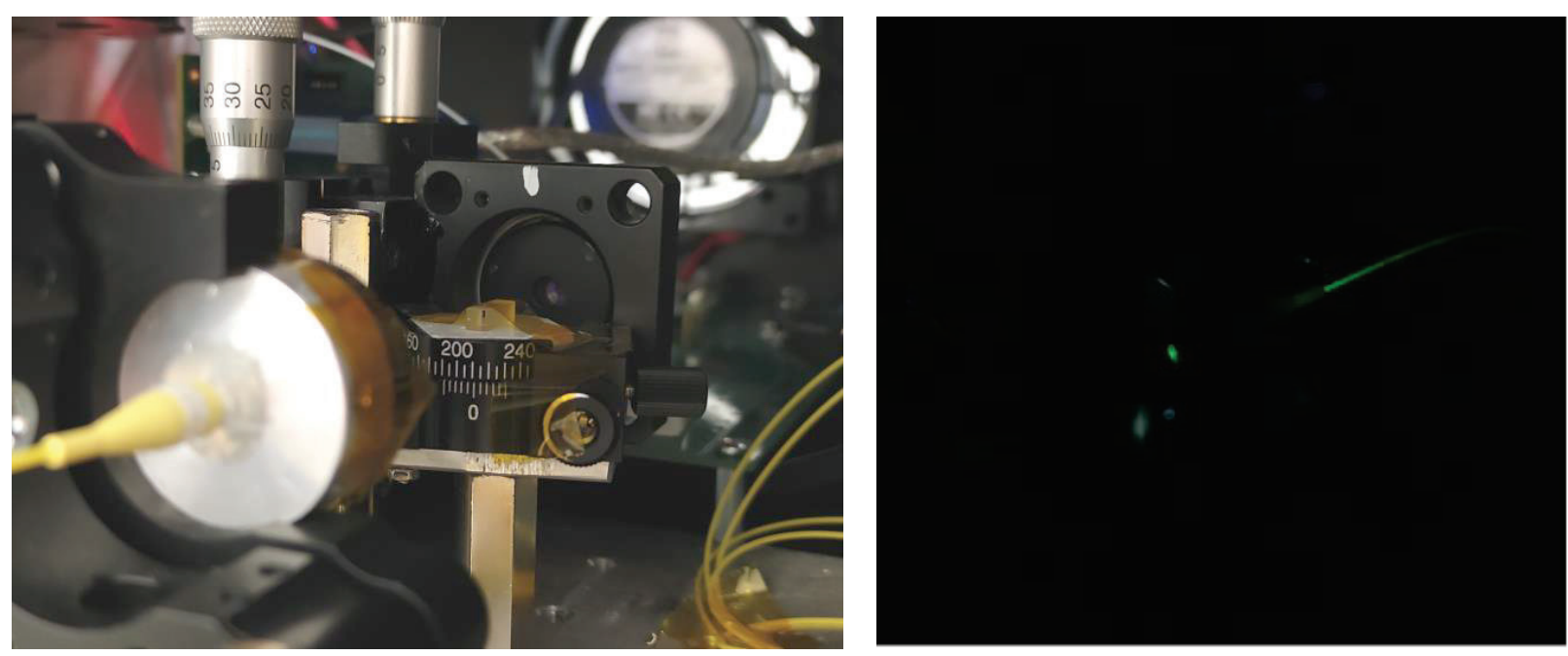

Figure 4. Set-up of SHG (left) and the beam at $532 \mathrm{~nm}$ passing through the VIS collimator (right).

Before the light hits the crystal, a collimation stage (PAF-X-2-C-FiberPort, Thorlabs) was used to achieve a higher power at output. Once the beam at $532 \mathrm{~nm}$ was obtained, the residual pump light was removed through a filter (FESH0550, Thorlabs). Finally, the beam was focused into the single mode fiber with another collimator (CFS2-532-FC, Thorlabs) for further signal attenuation to reach the requirements of the project. To balance the power amount in each echo similar process as with the NIR stage was followed. A variable voltage-controlled attenuator (V450F, Thorlabs) was used to adjust the desired power once the output was calibrated to the maximum level for each echo. This attenuator has a maximum attenuation value of $>30 \mathrm{~dB}$, the operating bandwidth between $450 \mathrm{~nm}$ and $600 \mathrm{~nm}$, and the voltage operating range of 0 to $5 \mathrm{~V}$. Next, an optical coupler (TW560R1A1, Thorlabs) was used to divide that power into two parts $(99: 1)$. High power branch was used to generate the short echo and the low power branch was used to generate the long echo, both according to the requirements of the project. Again, some fixed attenuators were used to reach the required peak power levels in both echoes and the output can be seen in the Figure 4.

\subsection{Ultraviolet stage - Sum Frequency Generation (SFG)}

The challenge of developing this emulator was to obtain the short and long pulse at $355 \mathrm{~nm}$ wavelength. The SFG technique was used to obtain at $355 \mathrm{~nm}$ beam. Two periodically poled lithium niobate (PPLN) crystals are used because the PPLN is a highly efficient medium for nonlinear wavelength conversion processes. Usually, it is used for frequency doubling [7], difference frequency generation [8], sum frequency generation [9], optical parametric oscillation [10], and other nonlinear processes.

The Mixer module (SC19348-01-01, HC Photonics) was used to achieve the $355 \mathrm{~nm}$ beam at the output by introducing a $1064 \mathrm{~nm}$ beam as input. The input beam, which was connected to the input optical fiber (PM980) of the Mixer module, was travelling through the two PPLNs buried inside the ovens which temperatures were controlled with the TEC controllers. Temperature-tuning method was used since PPLN crystals have multiple poling period (PP) sections in each crystal, each with a different poling period, allowing different wavelengths to be used at a temperature of given crystal.

For the first PPLN, the optimal temperature was determined by adjusting the phase matching temperature of the SHG stage while controlling the optical power output with a silicon photodiode (SIL PD). The 1064nm beam was removed with a visible filter (FESH0550, Thorlabs). For this purpose, an automatic temperature scan with 0.1-degree steps was performed by a program in LabView. Once the optimum temperature of the first PPLN was reached, this value was fixed.

Afterwards, second PPLN crystal was used to generate the UV beam by applying SFG (inputs: $532 \mathrm{~nm}$ and $1064 \mathrm{~nm}$ beams). To optimize the phase matching temperature of the SFG stage, the same procedure was performed as with the SHG stage but the UV enhanced SIL PD was used in this case. Once the beam at $355 \mathrm{~nm}$ was obtained, it was introduced into a multimode fiber (M91L01, Thorlabs) and an UV filter (UG-11, Edmund optics) was placed before the fiber to 
remove the pump beams. The output beam at $355 \mathrm{~nm}$ measured by the CCD spectrometer can be seen in Figure 5 . Due to lack of the COTS splitters with necessary features this stage is producing only long or short echo at a time.

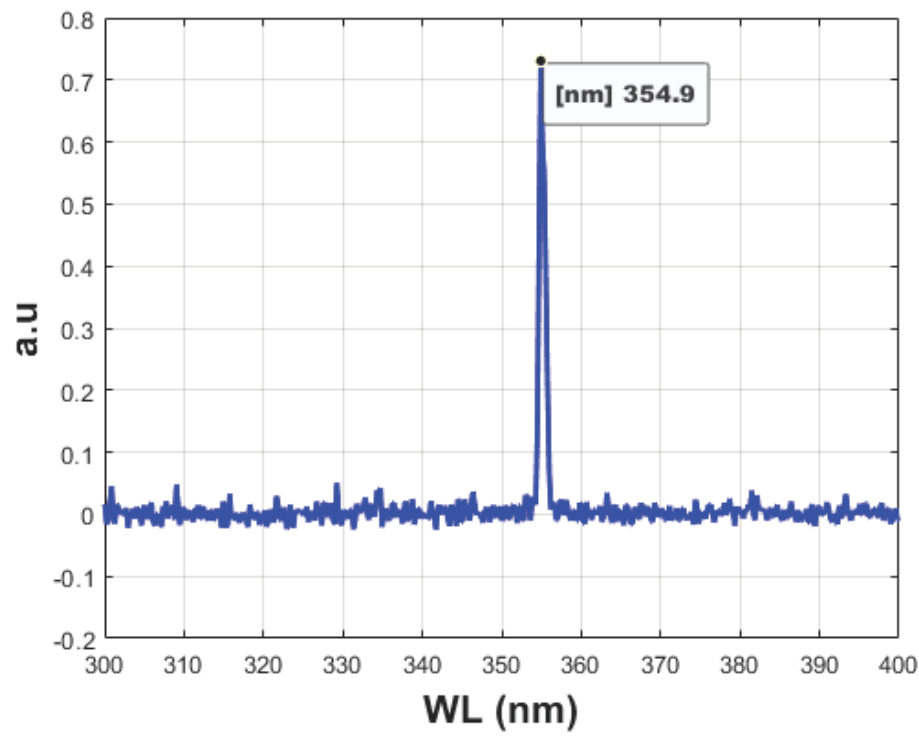

Figure 5 . The beam at $355 \mathrm{~nm}$ measured by spectrometer.

\section{TRANSPORT UNIT}

The complete device must be modular and relatively small to be put together with the remaining elements of the detection chain, as well as to provide mobility between the different partners of the project. Therefore, all components of the system were put inside the box with all necessary inputs and outputs available for characterization of the system, and further use in the dedicated application. Both, the interior and exterior of the system are shown in Figures 5 and 6 . It should be noted that inside the LEE there are two levels: the bottom one is used to fix the OPGS (Figure 7, right) and the VIS stage (Figure 7, center) whereas the top level is occupied by the NIR and UV stages (Figure 6, right). This system will help to characterize the critical optical parameters of a HgCdTe APD directly hybridized with a custom designed CMOS ROIC in the next measurement campaign (April 2021).
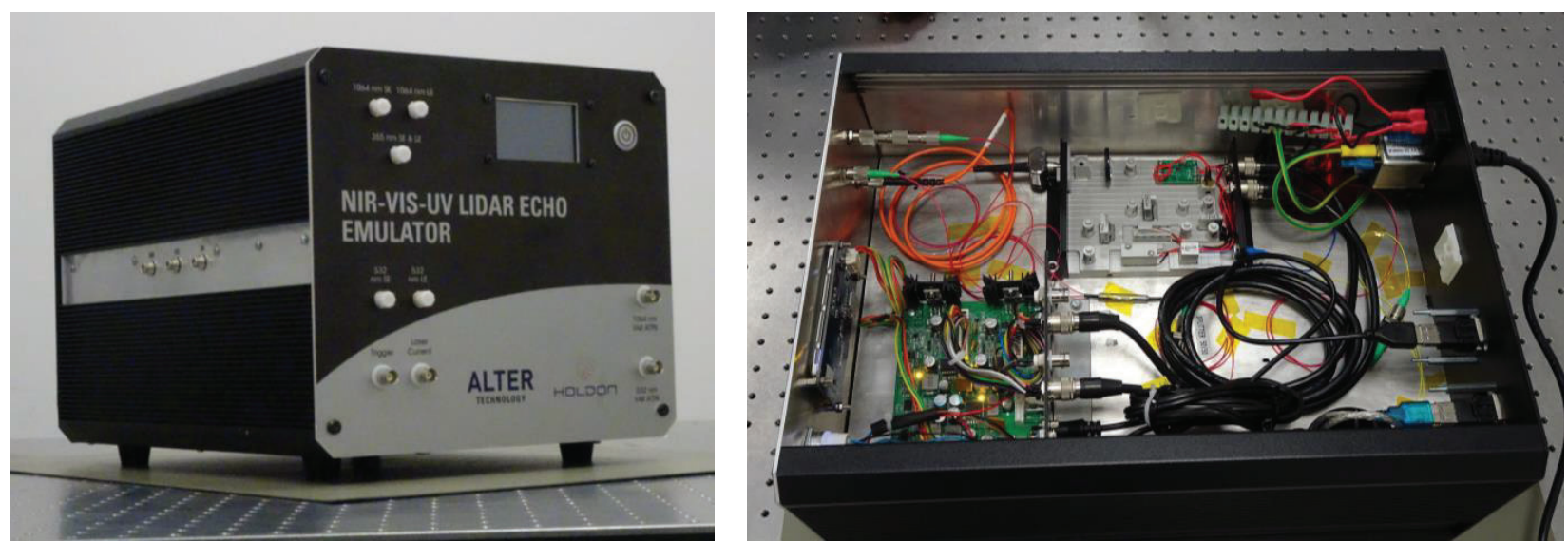

Figure 6. The transport unit of NIR-VIS-UV LEE (left) and on the top level inside the LEE (right). 

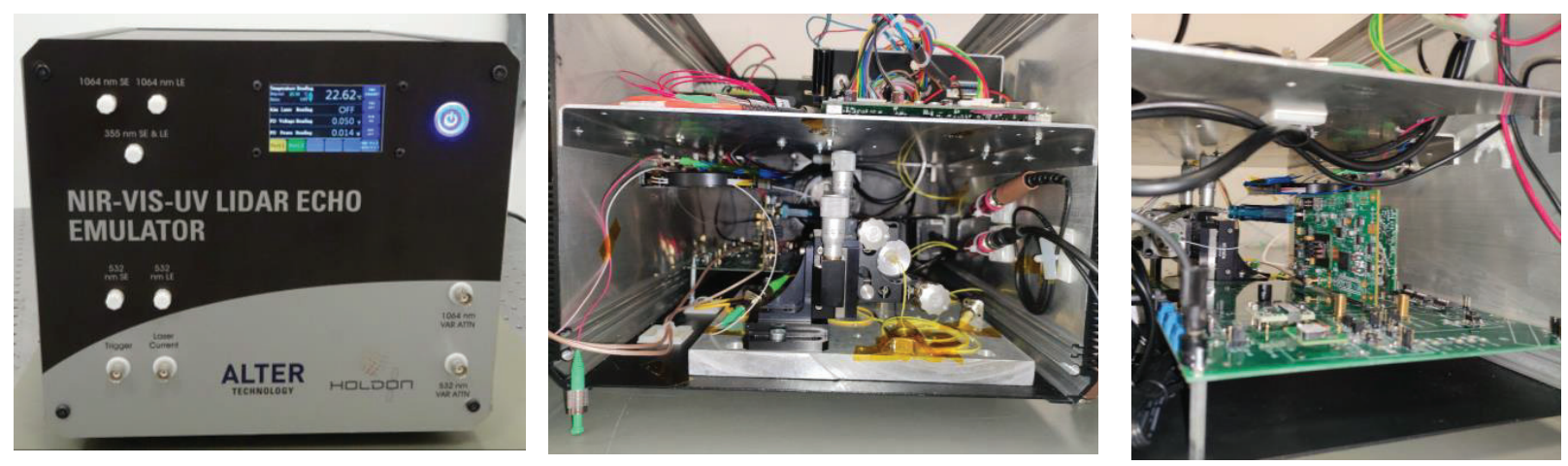

Figure 7. The front panel of NIR-VIS-UV LEE (left) and on the bottom level inside the LEE (center and right).

\section{CONCLUSIONS}

The LEE capable of emulating the echoes of a spatial lidar at three wavelengths $(355 \mathrm{~nm}, 532 \mathrm{~nm}$ and $1064 \mathrm{~nm})$ was developed. In general, it enables characterization of the critical optical parameters of the detection chain of a space or airborne lidar. The LEE allows overlapping short echo with long echo with $63 \mathrm{~dB}$ dynamic range. In-depth characterization of the lidar detection module will evaluate compliance with the requirements for which it was designed and to feed future designs with real figures from the lidar detection chain.

The demanding requirements of the European H2020 HOLDON project have been met regarding peak power, pulse shape, pulse width, dynamic range.

Techniques as SHG and SFG, commonly used in light sources in continuous mode, have been used in pulsed mode with satisfactory results even in this low power application.

\section{ACKNOWLEDGMENTS}

This work was supported by the EU Comission in frame of H2020 HOLDON Grant agreement ID: 776390. The authors M.A Geday and X. Quintana are grateful to the economic contribution from the Communidad de Madrid and EU structural funds (FEDER): Programa de Actividades de I + D SINFOTON2-CM (S2018/NMT-4326). The authors M. Rodriguez-Cortina and M.A. Geday want to express their gratitude to the Communidad de Madrid for the IND2017/TIC7758 grant for Industrial $\mathrm{PhD}$ funding

The authors thank to Olivier Saint-Pe, Kevin Aubaret, and Paul Bereau for fruitful discussions and hints during the development of the LEE.

\section{REFERENCES}

[1] J. Rothman et al., 'HgCdTe APDs for time-resolved space applications', CEAS Space J., vol. 9, no. 4, pp. 507-516, Dec. 2017, doi: 10.1007/s12567-017-0169-1.

[2] J. Rothman, 'Physics and Limitations of HgCdTe APDs: A Review', J. Electron. Mater., vol. 47, no. 10, pp. 56575665, Oct. 2018, doi: 10.1007/s11664-018-6475-3.

[3] Johan Rothman et al., 'HgCdTe APDs detector developments at CEA/Leti for atmospheric lidar and free space optical communications', Jul. 2019, Proc. SPIE vol. 11180, doi: 10.1117/12.2536055.

[4] Pawel Adamiec, Alvaro Machon, Monica Rodriguez Cortina, Alejandro Lopez Moya, Enrique Cordero, and Juan Barbero, 'LIDAR echo emulator', Jul. 2019, Proc. SPIE vol. 11180, doi: 10.1117/12.2535985.

[5] 'HOLDON'. https://holdon-h2020.eu/ (accessed Feb. 10, 2021).

[6] V. G. Dmitriev, G. G. Gurzadyan, and D. N. Nikogosyan, 'Properties of Nonlinear Optical Crystals', in Handbook of Nonlinear Optical Crystals, V. G. Dmitriev, G. G. Gurzadyan, and D. N. Nikogosyan, Eds. Berlin, Heidelberg: Springer, 1999, pp. 67-288. 
[7] S. Guo, J. Yang, B. Yang, T. Zhang, and J. Wang, 'Frequency doubling of 1560nm diode laser via PPLN and PPKTP crystals and frequency stabilization to rubidium absorption line', Quantum Nonlinear Opt., vol. 7846, p. 784619, Nov. 2010, doi: 10.1117/12.871688.

[8] K. Krzempek, G. Soboń, G. Dudzik, J. Sotor, and K. M. Abramski, 'Difference frequency generation of Mid-IR radiation in PPLN crystals using a dual-wavelength all-fiber amplifier', in Nonlinear Frequency Generation and Conversion: Materials, Devices, and Applications XIII, Feb. 2014, vol. 8964, p. 89641U, doi: 10.1117/12.2040925.

[9] Yingxin Bai et al., 'The application of PPLN sum frequency generation in mid-infrared detection', in 2005 IEEE LEOS Annual Meeting Conference Proceedings, Oct. 2005, pp. 96-97, doi: 10.1109/LEOS.2005.1547887.

[10] P. E. Britton et al., 'Optical parametric oscillation in periodically poled lithium niobate driven by a diode-pumped Q-switched erbium fiber laser', Opt. Lett., vol. 23, no. 8, pp. 582-584, Apr. 1998, doi: 10.1364/ol.23.000582. 